University of Nebraska - Lincoln

DigitalCommons@University of Nebraska - Lincoln

USDA National Wildlife Research Center - Staff Publications
U.S. Department of Agriculture: Animal and Plant Health Inspection Service

2012

\title{
Designing a monitoring plan
}

Eric M. Gese

USDA/APHIS/WS National Wildlife Research Center, eric.gese@usu.edu

Hilary S. Cooley

USDA National Wildlife Research Center, hilarycooley@gmail.com

Frederick F. Knowlton

Utah State University, knowlton@cc.usu.edu

Follow this and additional works at: https://digitalcommons.unl.edu/icwdm_usdanwrc

Gese, Eric M.; Cooley, Hilary S.; and Knowlton, Frederick F., "Designing a monitoring plan" (2012). USDA National Wildlife Research Center - Staff Publications. 1139.

https://digitalcommons.unl.edu/icwdm_usdanwrc/1139

This Article is brought to you for free and open access by the U.S. Department of Agriculture: Animal and Plant Health Inspection Service at DigitalCommons@University of Nebraska - Lincoln. It has been accepted for inclusion in USDA National Wildlife Research Center - Staff Publications by an authorized administrator of DigitalCommons@University of Nebraska - Lincoln. 


\section{6}

\section{Designing a monitoring plan}

Eric M. Gese, Hilary S. Cooley, and Frederick F. Knowlton

Monitoring is the collection and analysis of repeated observations or measurements to determine whether a management action is having the desired effect of meeting management objectives and demonstrating success or failure of a management strategy (Elzinga et al. 2001). Monitoring is composed of a series of surveys (sensu Chapter 2) framed in a design aimed at answering specific management questions. There are many reasons to establish monitoring plans, such as when a carnivore species is of a high social or economic value, is rare and decreasing in numbers, is in eminent danger of extinction, or is part of a legally mandated planning process. Monitoring is commonly conducted in combination with a formal research program with ecological objectives to provide managers and policy makers with information for making informed decisions and formulating conservation plans with some level of certainty or success (Nichols and Williams 2006; Sauer and Knutson 2008; McComb et al. 2010). Monitoring can also be useful for adaptive management strategies by treating management as a hypothesis and incorporating learning into the process with the data collected providing feedback about the effectiveness of alternative actions (McComb et al. 2010).

Designing a monitoring plan involves identifying the goals of the associated management plan, developing key questions, and designing a rigorous sampling scheme. Analyses must be pertinent to management objectives and capable of assigning probabilities to observed trends. Finalizing a monitoring design is a precursor to initiating data collection. Some monitoring programs fail to provide the information needed due to unclear or unspecific objectives, flawed or poor study design, low statistical precision or power to detect change, inconsistent commitment to implement or adjust the monitoring plan, or failing to communicate results to stakeholders (Elzinga et al. 2001).

This chapter provides the conceptual framework for designing a monitoring program with special emphasis on carnivores, but details on surveys that are 


\section{I Carnivore Ecology and Conservation}

part of monitoring programs are covered in Chapter 2. Elzinga $e t a l$. (2001) and McComb et al. (2010) describe the design and implementation of monitoring programs in more detail. Details of field techniques are covered in Chapters 4, 5, 6, 7,12 , and 13 .

\subsection{Identifying questions and monitoring designs}

To design a monitoring program, one must understand the biological system to be monitored and know the gaps in knowledge. Background knowledge is needed to articulate questions clearly; questions that ensure the data collected will be adequate to address the questions, fill knowledge gaps, test assumptions, and able to identify thresholds for altering management actions. Detail and focus are important at this stage. Use of vague or unclear terms, overly broad or ambiguous questions, and ill-defined spatial and temporal scales increase the risk data collected will not adequately address the key questions at scales that are meaningful. Questions that guide a monitoring program must be anchored to the objectives of the associated management plan. The questions must address the gaps in information about the-target-population-that-prevent-managers-from-understanding-how-the target population is responding to management actions, or predicting how the target population will respond to proposed future management actions. Many monitoring programs are set within a research program, allowing the key questions to be stated as hypotheses or as a number of alternative hypotheses. If the management plan dictates that managers need to know if the target population is increasing, then this need becomes a question for the monitoring program.

The four basic monitoring designs (McComb et al. 2010) address monitoring questions of different complexity.

1. Incidental observations are opportunistic observations of animals or sign. These are usually of little use within a monitoring framework except, perhaps, to provide preliminary information to a more structured plan.

2. Inventory designs document the presence or absence of the target species in an area (often referred to as a survey, sensu Chapter 2). The rarity of the species and the level of confidence in determining presence/absence are critical.

3. Status and trend monitoring (aka surveillance) designs establish trends over time by monitoring populations over long time-spans. The design of a monitoring plan should consider the scope of inference. Monitoring may be needed only for a local population, or may cover a large portion of the target species' geographic range and require participation by multiple agencies. For monitoring trends, sampling intensity must be designed to detect 
change, or lack of change, over time on the appropriate spatial scale. Chapter 2 provides background on sampling design.

4. Cause and effect monitoring designs allow evaluation of short- or long-term effects of a management action on a population and include such approaches as retrospective comparative mensurative designs or Before-After ControlImpact (BACI) designs (Stewart-Oaten et al. 1986; Gotelli and Ellison 2004).

\subsection{Developing a monitoring program}

Chapters 2, 4, and 8 outline the critical aspects of setting boundaries, selecting indicators to measure, developing sampling design, choosing sampling units and sites, calculating effect size, and choosing statistical analyses. In addition, developing a useful monitoring plan usually requires the simultaneous consideration of several major issues, each with embedded components. The monitoring plan must include techniques that are biologically appropriate and feasible, legally and socially acceptable, and must provide useful results with the resources available. Many potential problems can be avoided by careful thought during the design phase and asking advice from research and managerial personnel working in similar environments. Issues intuitive to experienced biologists may not be to a naive biologist.

Monitoring programs must stay within their budgets. If the optimal sampling design and sampling methods preclude meeting budget constraints, the monitoring questions, and perhaps the objectives of the management plan, need to be reevaluated. Developing a management program that cannot be carried out is a waste of time and money.

Gaining the necessary permits from governments and agencies involved in the area is an important hurdle to resolve early in the planning process. Often, such entities need to be consulted and even involved in the study design. Terms and methodologies should be clearly defined in research protocols and proposals to avoid confusion. If samples are to cross international boundaries, special permits may be required for export. Depending upon the capture methodologies involved, knowledge of, and permission to, handle non-target species must also be obtained. Procedures for handling target and any non-target species should be outlined. Completion of an approved handling and immobilization course from a qualified veterinarian should be considered. Some countries or agencies require a veterinarian be present when animals are captured, immobilized, and handled.

The ability to conduct a monitoring program could be curtailed if the social, political, or cultural values prohibit either the presence of you or your equipment. Cultural and social sensitivities related to the animals should be respected, 


\section{Carnivore Ecology and Conservation}

particularly where the local populace retains religious or cultural ties to the carnivore involved.

The manner in which individuals of a species distribute themselves across a landscape in both time and space is an important issue. When designing a monitoring plan, several issues should be considered: (1) Whether the animal is solitary or gregarious; for example, packs of animals are more readily sighted than solitary individuals thus influencing the probability of detection. (2) Does recognition of one individual influence recognition of others? Often for social canids, finding sign of one individual indicates the presence of others in the vicinity. (3) Is the interest focused toward assessing individuals or groups (e.g. packs or clans)? For some estimates, knowing a pack or social group is present may be sufficient for monitoring; while pack size may be necessary in other situations. (4) Whether seasonal movements, such as migrations or movements among different habitats, are apt to be involved and do they apply equally to all sex and age classes? (5) Whether the species is territorial, which may result in them being distributed in some regular fashion, and if territorial, how large are the territories and how does this relate to the size of area for which the assessment is being attempted?

Equally important in designing a program are attribures of the study area. The physical attributes, including size, topography, and nature of the environment play a role in determining what sort of activities are feasible and practical. This starts with a clear designation of the area or areas for monitoring with clearly defined boundaries. This is essential if complete enumeration is feasible or whether constraints on time or resources dictate some type of sampling. Size of area would be an important aspect but topographic and vegetative features would also be involved. Ultimately, the demarcation of the boundary of the population area to be assessed would be critical if an estimate of species' density is needed. Some carnivores occupy rough terrains, dense habitats, extreme habitats, roadless areas, or high elevations. The terrain can be used to an advantage. Placing remote cameras in situations where animals funnel down trails into a valley or through a mountain pass allows concentrating sampling efforts and increasing success of "capture." Stratifying track sampling along trails commonly traveled by the species may increase probability of detection. Prominent landscape features used by carnivores for scrapes or scent-marking can be also useful for sign surveys. Techniques that increase detection, however, may introduce bias for many sampling designs (Chapters 2, 4, and 8).

Identifying when to collect data will determine not only the merits of the information obtained, but also the inferences made from the data. Several issues dealing with the timing of sampling should be considered: (1) Seasonal changes in the activity or visibility of the animals. (2) Whether a seasonal pattern of 
population phenology is involved, when might sampling be best accomplished, and how does that relate to the question? (3) Among species with seasonal breeding patterns, characterizing the breeding population may be more important than making assessments at times that will include young of the year. (4) Among many species, dispersal patterns must be considered as they relate to naive animals moving across unfamiliar landscapes with consequent changes in population structure. In instances where some measure of reproductive performance is desired, conducting the assessments at the proper time of year may be required (if young animals can be discriminated from adults). The activity periods of many species are influenced by the prevailing weather or even lunar patterns.

Whenever possible, use typical behaviors or products of behaviors, to detect animals or assess species' abundance rather than elicited responses. Elicited responses may be influenced by social status or environmental conditions. For some species, especially when documenting "presence" is the primary objective, sampling can take advantage of stereotypic activities. Many felids, for example, have an affinity for traveling within narrow canyons or along specific ridge-tops. Similarly, since the distribution of black-footed ferrets (Mustela nigripes) appears limited primarily to prairie dog (Cynomys spp.) towns, it is reasonable to limit assessments to such areas. During development of inventory procedures for coyotes (Canis latrans), the use of elicited vocalizations to assess abundance was considered (Okoniewski and Chambers 1984). Early trials determined that a four-fold difference in response rates resulted from three different types of sirens used to elicit the vocalizations, and coyotes were likely to respond at times they were active but unlikely to respond when they were inactive. Wolfe (1974) reported that while dominant (alpha) individuals were most likely to respond, transient individuals were appreciably less likely to respond. While taking advantage of such behaviors can increase sampling efficiency, researchers need to accommodate for potential biases that unequally represent specific sex, age, and social classes.

Many carnivore species have an innate curiosity to novel situations in their environment. As the objects become familiar through repeated exposures, they elicit less interest. Sometimes simply moving the stimulus a small distance will revive interest; however, new or rearranged objects can induce neophobic responses. Sensitivity to such situations varies widely among species. While coyotes react strongly, and warily (neophobia), to novel stimuli (Windberg and Knowlton 1988; Windberg 1996; Harris and Knowlton 2001), bobcats (Lynx rufus) are much less reactive to novel situations and can be repeatedly trapped, even in the same locations with the same attractants. Knowledge of the repertoires of species can be important for selecting sampling methods. 


\section{I Carnivore Ecology and Conservation}

Statistical hypotheses are widely used because they provide objective, standardized criteria for decision-making. However, this has received much criticism over the last decade Oohnson 1999, 2002; Anderson et al. 2000; Burnham and Anderson 2002; Ellison 2004; Guthery 2008). Null hypothesis testing is uninformative in some cases (Johnson 1999), and often results in conclusions that lack meaningful insights for conservation, planning, management, or further research (Guthery 2008). Additionally, the significance level (a) used in a test is often based on convention (i.e. $a=0.1,0.05$ ), classifying results into biologically meaningless categories (significant and non-significant) (Anderson et al. 2000). There may be times when a biologist, faced with a test statistic with a $p$-value of $>0.05$ but $<0.10$, may decide that a result is biologically meaningful or suggestive of a relationship. Bayesian and information-theoretic approaches are often more applicable for analyses in monitoring programs than traditional parametric, or even nonparametric statistical techniques. Learn the analytical techniques most appropriate for the monitoring program.

\subsection{Evaluating the monitoring plan}

After data has been collected and analyzed, biologists and managers must decide: given the information, what should we do? Several alternatives can be considered: (1) continue to monitor, (2) use the information to make changes in the management programs, as well as the monitoring plan, (3) evaluate the risk of changing versus continuing with the status quo, and (4) determine if integrating the data with data from other programs will produce a broader picture of the species or system (McComb et al. 2010).

\subsubsection{Thresholds and trigger points}

Within any monitoring program there are a multitude of issues to be addressed by managers and stakeholders before making any changes in the plan. One suggested approach is to agree with the stakeholders at the outset that if a particular threshold or trigger point is reached, alternative management actions need to be implemented (McComb et al. 2010). Trigger points might be considered points initiating a change to a management program, whereas thresholds indicate success in a management action (Block et al. 2001). Preferably, stakeholders have agreed beforehand to a series of steps to be taken, if a trigger point is reached. A potential problem with thresholds is they may result from social negotiation among stakeholders, and define a socially and mutually acceptable level of progress that may not be biologically dependable (McComb et al. 2010). 


\subsubsection{Forecasting trends}

With several years of data, trends may emerge providing information to guide management actions. However, one must remember that the degree of precision decreases, the further that forecasts are predicted into the future, so forecasting trends beyond the dataset should be viewed cautiously and serve as one tool in guiding management decisions. Variation associated with trends and trend analyses, especially for tare species, is often high and the power associated with detecting a significant trend is often low.

Computer simulations have been used to model carnivore populations under diverse conditions (e.g. Connolly 1978; Mowbray et al. 1979; Lindzey and Meslow 1980; Sterling et al. 1983; Pitt et al. 2003; Conner et al. 2008). These models can be used to simulate population responses when one or more demographic variables are manipulated. Trigger points can be established from the risk assessment, prompting alternative management actions. Examining the sensitivity of the model to changes in the initial conditions of the system, parameter values, and structural features of the equations is useful for model assessment (Williams et al. 2002b). Population viability analysis (PVA) and population and habitat viability assessment (PHVA) can be used to evaluate the outcomes of various management actions, environmental perturbations, and stochastic events on the population viability of a species over a predetermined period of time (Shaffer 1981; Boyce 1992; Reed et al. 1998). Biologists using such models should consider the "realism" of the models and should ensure that the models are adaptive in response to ecological, environmental, and management factors (Williams et al. 2002b). A PVA or PHVA is only a model and is only as valid as the assumptions and information upon which they are based. They may not reflect or predict population persistence, and should not be the primary tool for developing conservation plans. Macdonald et al. (1998) suggest that PVAs may be most useful to biologists for developing and guiding management actions and identifying practical monitoring methods. Always evaluate the accuracy of the data incorporated and the levels of uncertainty (Reed et al. 1998; Williams et al. 2002b). Some PVAs and PHVAs may be used to raise questions and formulate hypotheses for future testing (Macdonald et al. 1998; Reed et al. 1998; Williams et al. 2002b).

\subsubsection{Predicting patterns over space and time}

Biologists and managers like to know where on a landscape a species is likely to occur, so management actions might increase or decrease populations, or might have minimal effects on the target species (McComb et al. 2010). Monitoring the presence of carnivores across a landscape provides information on the spatial 


\section{0 | Carnivore Ecology and Conservation}

distribution of individuals within populations, and provides a better understanding of meta-population structure and connectivity among subpopulations. If demographic rates are known, the value of subpopulations as sources or sinks can be examined (Chapter 10), as well as the probability of subpopulations becoming locally extinct and subsequently recolonized.

\section{6:3.4-Integrating monitoring data}

Data from a monitoring plan can be integrated with other environmental data to produce an integrated view of a landscape, thereby allowing managers to evaluate individual parts, as well as the whole landscape (McComb et al. 2010). These approaches use data to parameterize a spatial and temporal model to increase understanding of possible future conditions on the landscape. This allows for examination of various "what if" scenarios for comparing alternative actions. In addition, the approach can identify key parameters to be monitored in the future to help stakeholders understand whether the results of a management action are being realized. The danger of using these models is that they may not have been tested with independent data and, therefore, their accuracy is completely unknown. The potential for wildly incorrect management actions is very real (Chapter 11).

\subsubsection{Risk analysis}

Risks from environmental stressors, disturbances, or hwman activities may be important when evaluating a management plan. Monitoring data can be used in risk analysis in a stepwise process to assess threats (e.g. Hull and Swanson 2006). Risk assessment is a procedure to determine threats and understand uncertainty providing an estimate of the likelihood and severity of species, population, or habitat loss or gain, and an evaluation of the potential tradeoffs associated with various management actions (McComb et al. 2010). Kerns and Ager (2007) proposed a quantitative and probabilistic risk assessment to provide a bridge between planning and policy.

\subsection{Changing the monitoring plan}

Extensive time and money are expended in executing a monitoring plan. Consequently, the design of these programs must be scientifically and statistically rigorous, and managers and stakeholders must understand exactly how the information will be used to make decisions (McComb et al. 2010). Decisions should be made using a sequence of steps: characterize the problem or question, identify the full range of alternatives, determine a set of criteria for selecting one, collect information about each option and evaluate it based on the criteria. Then make 
a decision. Data collected in an adaptive management framework should use the information gained to refine the monitoring plan and improve the quality and utility of the data (McComb et al. 2010).

Changing a plan has consequences that must be considered carefully. If the data collected and analyzed suggest the goals and objectives are not being adequately met, changes to the plan may be required. Adding or dropping variables to be measured may be required as information reveals new patterns or processes, or budget constraints necessitate reducing the number of variables that can be measured. Changing the location or periodicity of sampling, or attempting to increase precision in data collection, are changes that can be considered. Avoid making changes that cause some or all of the data already collected to be incompatible with data collected after the change (McComb et al. 2010). Changing a monitoring program should not be done lightly and necessitates as much preparation as establishing the initial plan. Gaining information and revising management approaches based on that new information is the main objective of monitoring. If changing conditions preclude managers following the sampling design and, therefore, the questions addressed by the monitoring program and the objects of the management plan cannot be met, the management plan and its objectives need to be reconsidered (Chapter 2).

Deciding when to terminate a monitoring program is equally difficult. Generally the decision to terminate monitoring should be based upon whether the questions associated with the objective of the management plan have been answered. The decision of when to end the program should be outlined in the monitoring plan itself (Chapter 15). If the data collected through the monitoring plan indicate a carnivore population has been increasing over the last 4-5 years and may be reaching carrying capacity, and this is the main objective of the program, then terminating monitoring may be a logical step. If the data indicate a declining population and the key questions have not been answered, the main objective of the program has not been attained and continuation of data collection may be necessary. 